\title{
Predissociation of $\mathrm{O}_{2}$ in the $\mathrm{B}$ state
}

\author{
A. M. Wodtke, L. Huwel, H. Schlüter, H. Voges, G. Meijer, and P. Andresen
}

Max Planck Institut fuer Stroemungsforschung, Bunsenstr. 10, 3400 Goettingen, West Germany

(Received 14 March 1988; accepted 13 May 1988)

\begin{abstract}
Dispersed LIF spectra of $\mathrm{O}_{2}$ in the Schumann-Runge band were measured with a modified tunable ArF laser in a flame. Spin-state selective predissociation of the $B$ state was directly observed in fluorescence excitation spectra, revealing the relative coupling matrix elements of the triplet components to the manifold of repulsive states. Such data determines the symmetries of the important predissociating curves for each observed $B$-state vibrational level and shows that past interpretation of absorption linewidth data is in error. Due to the fast predissociation, quench-free emission spectra arising from laser prepared single rovibronic levels in the $B$ state were observed even in an atmospheric flame. Fluorescence to $X$-state vibrational levels as high as $v^{n}=35$ was observed and relative emission probabilities were derived.
\end{abstract}

\section{INTRODUCTION}

The spectroscopy of $\mathrm{O}_{2}$ in the Schumann-Runge band is one of the most extensively studied topics in diatomic spectroscopy. ${ }^{1,2}$ The importance of $\mathrm{O}_{2}$ in solar absorption, atmospheric photochemistry, and combustion, as well as being a colorful example of molecular structure, has provided the motivation for the extensive effort given to this problem.

The Schumann-Runge band is photochemically very important because of the predissociation of the $B$ state. $A b$ sorption linewidth measurements have shown vibrational and rotational state dependent lifetimes for $v^{\prime}=0-19$ and $J^{\prime}=0-20$, varying from 2 to $25 \mathrm{ps}^{3}{ }^{3}$ Such data has been the subject of extensive theoretical effort. Spin-orbit coupling of the $B$ state to a manifold of repulsive states is the dominant predissociation mechanism. ${ }^{4}$ The vibrational state dependence for the $J=0$ states has been fit and interpreted as the result of four repulsive curves, each with different vibrational state dependent predissociation efficiencies. ${ }^{3.5}$ According to this analysis, the ${ }^{5} \Pi_{u}$ repulsive curve, which is also responsible for the observed spectroscopic perturbations analyzed by Julienne, ${ }^{5}$ is dominant for nearly all vibrational levels. The other three $\left({ }^{1} \Pi_{u},{ }^{3} \Pi_{u}\right.$, and $\left.{ }^{3} \Sigma_{u}^{+}\right)$are thought to play a minor but nonnegligible role and are predicted to produce no spectral perturbations, observable with available resolution. ${ }^{5}$ The rotational state dependence has been fit assuming a simple centrifugal distortion model within the spin-orbit dissociation mechanism. ${ }^{3}$ No evidence of orbitrotation interaction is seen in the rotational dependence.

Despite the good theoretical fit to the results of absorption spectra, one must view this apparently well-treated problem with suspicion. The spin-orbit operator represents an interaction of the magnetic dipole moment of the electron spin with a magnetic field due to electronic angular momentum. An interaction between different degrees of freedom of electronic motion may not produce clearly interpretable rotational and vibrational dependent predissociation lifetimes. The clearest effect should appear in the spin-state dependence of the lifetimes. Such experimental results are not yet available for $\mathrm{O}_{2}$ from absorption spectra.

Dispersed LIF spectra of $\mathrm{O}_{2}$ exist in only a few cases, because of the very low fluorescence quantum yield and the lack of intense light sources in the wavelength region of the Schumann-Runge band. ${ }^{6}$ This is however a much better way to investigate the state dependent predissociation of $\mathrm{O}_{2}$ in the $B$ state. By dispersing the fluorescence, the spectra can be simplified and as will be shown the triplet can be resolved. In LIF there is no need to make linewidth measurements because the signal intensities are directly proportional to the predissociation lifetimes.

In this experiment strong LIF spectra of $\mathrm{O}_{2}$ have been observed using a tunable ArF laser. The wavelength scanning range of this laser is sufficient to interrogate transitions to $v^{\prime}=10,11,12,14,15,16$, and 17 . For $v^{\prime}=11,14,15$, and 16 , the resolution of the laser was sufficient to resolve the components of the spin multiplets. This provides a very clear picture of the predissociation mechanism in $\mathrm{O}_{2}$.

The high pumping efficiency of the laser has also allowed the observation of dispersed emission spectra of $\mathrm{O}_{2}$ originating from $v^{\prime}=10,11,14,15$, and 16 and terminating in states as high as $v^{\prime \prime}=35$. The relative emission probabilities between vibrational states of the $B$ and $X$ curves have been extended nearly to the dissociation limit of the $X$ state.

\section{EXPERIMENTAL}

Figure 1 illustrates the experimental arrangement. In order to populate the states of interest, $\mathrm{O}_{2}$ was observed near the edges of a flame, produced by a butane soldering torch that was borrowed from the workshop and used without modification. There are no collisional effects in the experiment because the predissociation rate of the $B$ state is much faster than the intermolecular collision rate in the atmospheric flame. This can be seen clearly in the emission spectra shown below (Sec. IV). Laser induced fluorescence was produced by the unfocused output of a modified Lambdaphysik EMG150 tunable excimer laser operating with argon fluoride. The laser delivered light pulses $3 \times 1 \mathrm{~cm}$ in size, with $100 \mathrm{~mJ}$ of energy and a $1 \mathrm{~cm}^{-1}$ bandwidth. One part in 1000 of the LIF was collected by an $f / 5.3$ one-to-one imaging lens and was dispersed through a $0.3 \mathrm{~m}$ Macpherson 218 monochromator with a linear dispersion of $2.65 \mathrm{~nm} / \mathrm{mm}$ and $0.2 \times 10 \mathrm{~mm}$ slits. The estimated volume of the flame sampled by the imaging system of the monochromator was 


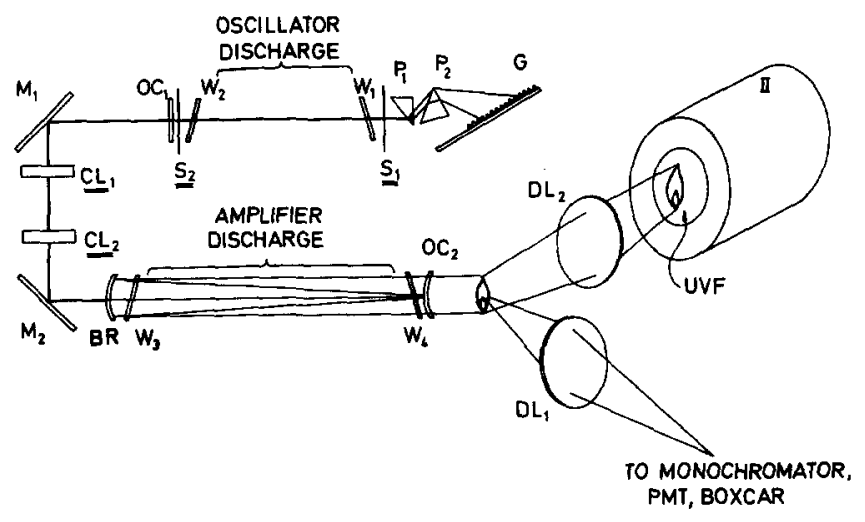

FIG. 1. Experimental apparatus with modified ArF laser: Grating (G), prisms $\left(\mathrm{P}_{1}, \mathrm{P}_{2}\right)$, windows $\left(\mathrm{W}_{1}, \mathrm{~W}_{2}, \mathrm{~W}_{3}, \mathrm{~W}_{4}\right)$, slits $\left(\mathrm{S}_{1}, \mathrm{~S}_{2}\right)$, mirrors $\left(M_{1}, M_{2}\right)$, output couplers $\left(C_{1}, C_{2}\right)$, cylindrical lenses $\left(C_{1}, C L_{2}\right)$, back reflector (BR), Detection lenses $\left(\mathrm{DL}_{2}, \mathrm{DL}_{2}\right)$, image intensifier (II).

$2 \times 0.2 \times 10 \mathrm{~mm}$. The dispersed fluorescence was detected by a RCA C31034 photomultiplier. Most spectra were averaged over three laser shots with a SRS boxcar whose $35 \mathrm{~ns}$ gate was centered around the laser pulse itself. No fluorescence was observed except during the laser pulse because all states of $\mathrm{O}_{2}$ predissociate faster than $25 \mathrm{ps}$. The monochromator was sufficient to remove all scattered laser light away from the lasing wavelength and the short gate discriminated against $\mathrm{cw}$ flame light to a level below the noise of the measurements. The spectra were simultaneously recorded by a strip-chart recorder and stored in the memory of an IBMPC.

Spectral assignments were determined from model spectra, synthesized with a program incorporating the molecular constants derived by Creek and Nichols ${ }^{2(a)}$ and the spinspin, spin-rotation constants of Bergeman and Wofsy. ${ }^{2(b)}$ In order to include the laser output as a function of wavelength, the narrow band output of the oscillator was measured at a $2.4 \mathrm{~m}$ distance, the distance between the laser output and the flame. Although this is only an approximate method for normalizing the observed spectra to the laser power, it does include in a near-quantitative way the variation of laser locking efficiency and the effect of atmospheric $\mathrm{O}_{2}$ absorption $\left(\mathrm{O}_{2} B^{3} \Sigma_{u}^{-} v^{\prime}=4-X^{3} \Sigma_{g}^{-} v^{\prime \prime}=0\right)$ over the laser scanning region. The transmission function of the monochromator (modeled as a triangle function) and the laser bandwidth are also simulated by the program. Line shapes for the $v^{\prime}=11 \leftarrow v^{\prime \prime}=2$ lines (see below) where Lorentzian line shapes were observable, were simulated using known vibrational state dependent linewidths and theoretically derived linewidths for the components of the triplet based on the theory of Julienne. ${ }^{5}$ Here the Lorentzian linewidths were calculated and a Gaussian convolution of the laser bandwidth was "numerically scanned" in a stepwise manner over the theoretical spectrum. All other lines have known Lorentzian linewidths much smaller than the laser bandwidth and for these, a Gaussian line shape with the half-width of the laser was calculated around each line.

After an initial period of success and then subsequent difficulties, the original Lambdaphysik (EMG150) design of the tunable ArF laser was modified to achieve a larger scanning range and better locking efficiency. The details and quantitative improvement achieved will be reported in a separate publication. ${ }^{7}$ The principal modification was to replace the $1.5 \mathrm{~mm}$ holes that define the active volume of the oscillator cavity with $1.5 \times 5 \mathrm{~mm}$ slits and to telescope the resulting rectangular output of the oscillator back to a $1.5 \times 1.5 \mathrm{~mm}$ spot using two cylindrical lenses. This improved not only the output power of the oscillator but also reduced the shot-toshot fluctuations that arise from instabilities in the discharge.

The shallow valley which underlies all the spectra shown below is an indication that the laser is operating well. On the edges of the scanning range where the broad band component of the excimer laser is a large part of its total emission, many $\mathrm{O}_{2}$ transitions are weakly excited and observable at the chosen emission wavelength. As the laser becomes well locked in the middle of the scanning range, this source of signal diminishes to about $7 \%$ of the resonant signal. A comparison of the background noise level on the edges and in the middle of the scanning region shows that the locking fluctuations are also smaller in the middle of the scanning range. The apparent spectral transition that appears in many of the spectra at $51790 \mathrm{~cm}^{-1}$ is due to atmospheric absorption by $\mathrm{O}_{2}$ in the laser's oscillator which at this wavelength causes the laser to operate completely broad band. The signal rises approximately to the level present on the edges of the scanning range and then returns to the locked mode when the atmospheric $\mathrm{O}_{2}$ absorption is passed by. Although there are four atmospheric absorptions in the laser scanning range, this effect is only observed at $51790 \mathrm{~cm}^{-1}$ where the output of the oscillator is already fairly weak.

\section{III. $\mathrm{O}_{2}$ LIF EXCITATION SPECTRA IN A FLAME}

$\mathrm{The}_{2} \mathrm{O}_{2}$ transitions that have been observed in excitation in a flame arising from $v^{\prime \prime}=2$ and 3 will appear elsewhere. ${ }^{8}$ We have observed only very weak transitions from $v^{\prime \prime}=0$ or 1. This is an expected result, due to the much smaller absorption cross sections and the faster predissociation rates of the accessible upper states at the ArF wavelength. ${ }^{9}$ Excitation of states above $v^{\prime \prime}=3$ goes directly to the $B$-state continuum and is expected to play no role in this experiment.

Figure 2 shows a typical LIF spectrum of $\mathrm{O}_{2}$ in a flame with a spectral simulation (above). The monochromator was adjusted to observe $v^{\prime}=14 \rightarrow v^{\prime \prime}=21$ emission at 351.5 $\mathrm{nm}$ with $0.5 \mathrm{~nm}$ resolution. This excitation spectra results primarily from the $v^{\prime}=14 \leftarrow v^{\prime \prime}=3$ transition, although $v^{\prime}=15,16 \leftarrow v^{\prime \prime}=3$ are also present. This signal to noise was obtained with a scan duration of about $10 \mathrm{~min}$. The relative intensities between rotational states could be fit with a temperature of $1750 \pm 250 \mathrm{~K}$. This is in agreement with previous temperature measurements of $\mathrm{OH}$ using a tunable $\mathrm{KrF}$ laser. ${ }^{6}$ Spatially dependent temperature measurements in Ref. 6 also showed that there is only a small temperature gradient in the region of the flame probed in this experiment.

The apparent doublets in the spectrum are due to the partial resolution of the $\mathrm{O}_{2}$ spin triplet in $v^{\prime}=14$. Each component of the triplet has the same $N$ quantum number (referring to nuclear rotor angular momentum). The red shifted transition always prepares the $B$-state $F_{1}$ component, 


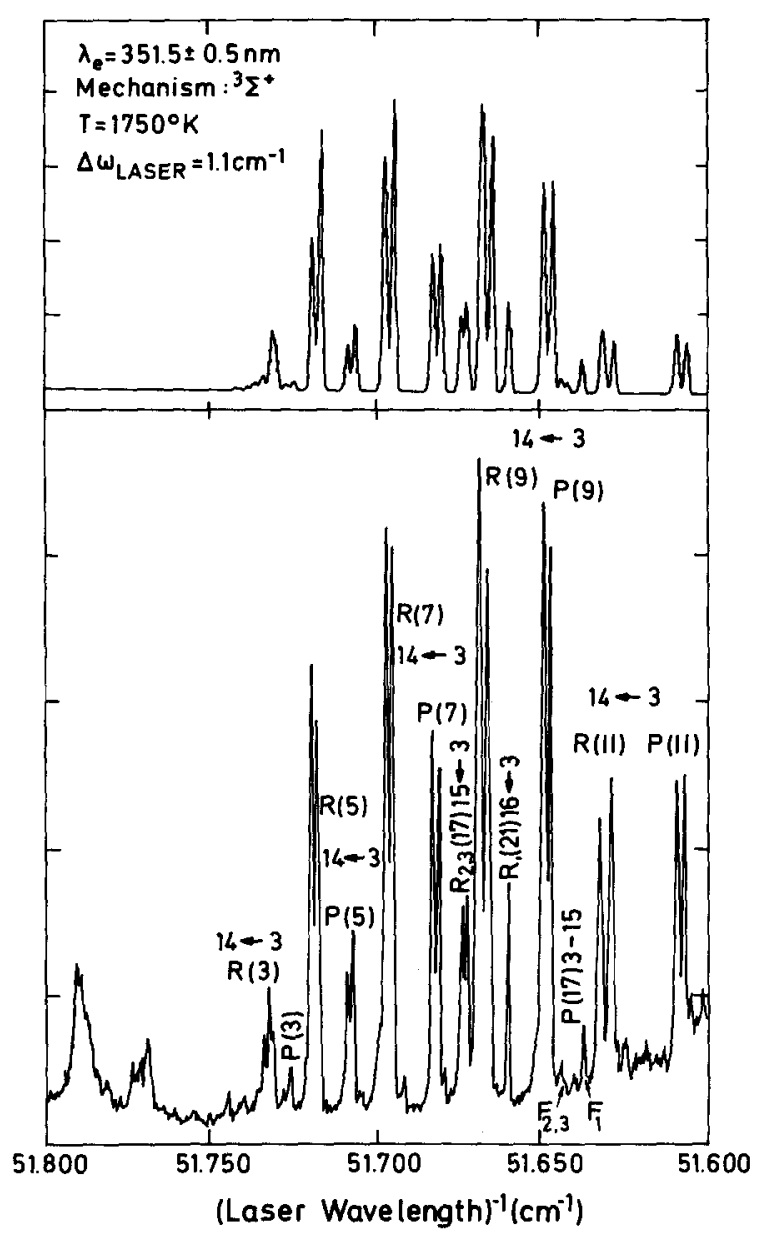

FIG. 2. Laser induced fluorescence spectrum: $v^{\prime}=14,15,16-v^{\prime \prime}=3$ simulated (above), measured (below).

$J=N+1$, and the blue shifted line contains both the $F_{2}$, $J=N$, and $F_{3}, J=N-1$, components. In Hund's case B, all three components have the same absorption probability, neglecting very small differences in the Hoenl-London factors and deviations for the lowest rotational states. Emission from the different components of the triplet occurs at almost the same wavelength and there is consequently no discrimination between the components due to the monochromator. In addition, the polarization of the emission coming from the three components is negligibly different even for the lowest $J$ states. It is therefore immediately clear that the three do not predissociate with the same probability. Empirically, one can conclude that the sum of the $F_{2}$ and $F_{3}$ lifetimes must be about equal the $F_{1}$ lifetime.

In Fig. 3, the triplet is fully resolved in several ( $B$ $v^{\prime}=15,16 \leftarrow X v^{\prime \prime}=3$ ) transitions due to the larger spinspin and spin-rotation constants for $v^{\prime}=15$ and 16 . Note particularly the well isolated $v^{\prime}=16 \leftarrow v^{\prime \prime}=3: \mathrm{R}(19)$ triplet at $51750 \mathrm{~cm}^{-1}$. Although a quantitative simulation of this spectrum is elusive due to its increased complexity, the qualitative observation that $F_{1}$ states predissociate approximately half as quickly as $F_{2}$ and $F_{3}$ states is clear for $v^{\prime}=14,15$, and 16.

The relative lifetimes of the triplet are directly related to the symmetry of the repulsive state involved, as will be

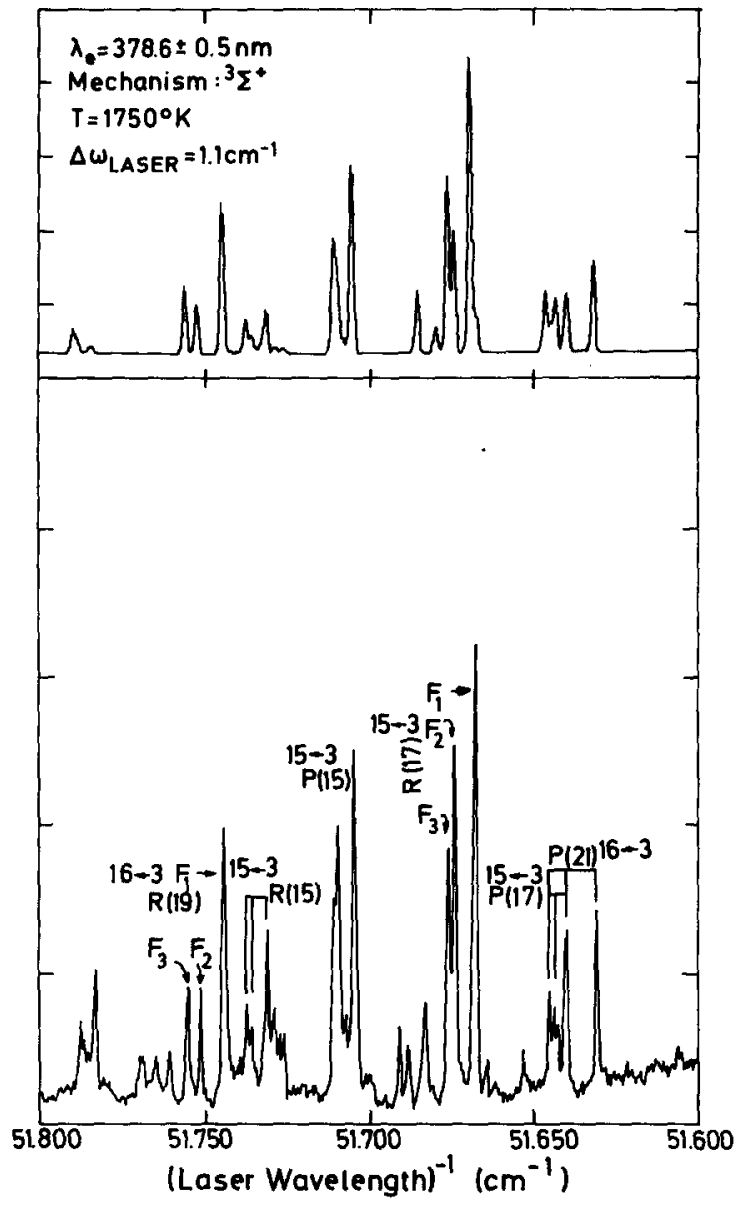

FIG. 3. Laser induced fluorescence spectrum: $v^{\prime}=15,16,17 \leftarrow v^{\prime \prime}=3$ simulated (above), measured (below).

shown in more detail below. From the relative coupling matrix elements calculated by Julienne ${ }^{5}$ one can conclude that $v^{\prime}=14,15$, and 16 are predissociated primarily by a state of ${ }^{3} \Sigma_{u}^{+}$or ${ }^{1} \Pi_{u}$ symmetry, whose triplet lifetime patterns are identical. While a ' $\Pi_{u}$ state has been predicted to be important for predissociation of low vibrational levels, no potential calculations have suggested that a curve of this symmetry could predissociate $v^{\prime}=14,15$, or 16 . However, a ${ }^{3} \Sigma_{u}^{+}$curve has been calculated to lie in the correct energy range and to have a fairly large spin-orbit coupling magnitude. ${ }^{10}$ The assumption of ${ }^{3} \Pi_{u}$ or ${ }^{5} \Pi_{u}$, the latter thought to be dominant from absorption spectra, implies completely different triplet patterns. For example in the case of the well isolated $v^{\prime}=16 \leftarrow v^{\prime \prime}=3: R(19)$ transition, the $F_{1}: F_{2}: F_{3}$ ratio's are 0.7:1.0:0.6 and 1.0:0.9:0.9, respectively, for ${ }^{3} \Pi_{u}$ and ${ }^{5} \Pi_{u}$. This shows the sensitivity of measuring spin-state dependent lifetimes as opposed to rotation vibration dependent lifetimes.

The observed importance of the ${ }^{3} \Sigma_{u}^{+}$curve urged us to investigate the $v^{\prime}=11$ level. Based on the interpretation of absorption spectra, this vibrational state should be where the ${ }^{3} \Sigma_{u}^{+}$repulsive curve has the largest influence observable in our experiment (although ${ }^{5} \Pi_{u}$ was still thought to be dominant for this level). Moreover, the $v^{\prime}=11$ state predissociates some 10 to 20 times faster than $v^{\prime}=14,15,16$ and rep- 


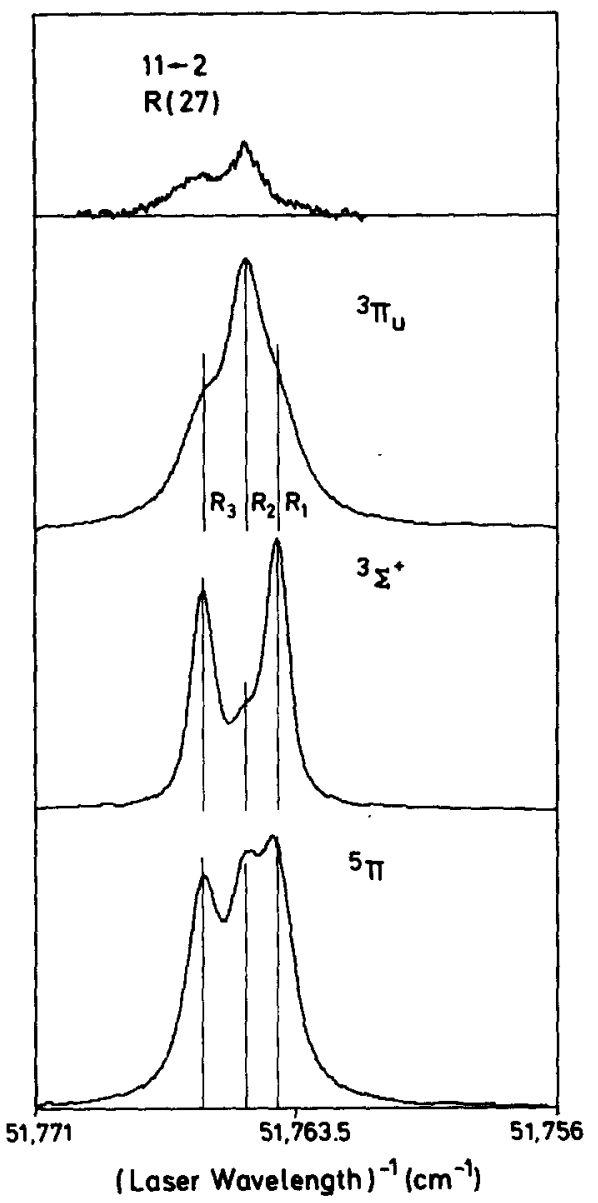

FIG. 4. High resolution laser excitation spectrum of $v^{\prime}=11-v^{*}=2$ : $\boldsymbol{R}(27)$ triplet. Simulated spectra assuming three different symmetries for the repulsive states. resents a much more important $\mathrm{O}$-atom production channel in the atmosphere. In an effort to achieve a clear picture of the predissociation mechanism of $v^{\prime}=11$, the laser was operated with a resolution of $0.5 \mathrm{~cm}^{-1}$ by fully illuminating the oscillator's grating and excitation spectra of well isolated $v^{\prime}=11 \leftarrow v^{\prime \prime}=2$ triplets were measured.

Figure 4 shows the excitation spectrum of the $R(27)$ triplet. The Lorentzian influence on the line shape is clearly visible, note the long wings on the lines. Three simulated spectra are also shown based on the state symmetry dependence of the relative coupling matrix elements from Ref. 5 . Only a ${ }^{3} \Pi_{u}$ repulsive state reproduces the observed line shape. Therefore, it is clear that it is the dominant mechanism. This is in stark contrast to theoretical predictions which have considered ${ }^{3} \Pi_{u}$ to be relatively unimportant. How LIF measurements of spin-state dependent lifetimes can lead to such different results than analysis of absorption spectra will be discussed below.

\section{DISPERSION SPECTRA}

Figure 5 shows two dispersed fluorescence spectra. The lower spectrum results from broad band excitation where the oscillator of the laser is blocked. The broad emission lines come from excitation of all accessible molecular transitions within the gain profile of the laser and subsequent emission from a large number of rotational states in $\mathrm{O}_{2} v^{\prime}=10,11,12$, $14,15,16$, and 17 as well as a small amount of NO $D$-state emission at $208.9 \mathrm{~nm}$. " Narrow band excitation of two coincident $\mathrm{O}_{2}$ rovibronic transitions $\left[v^{\prime}=10 \leftarrow v^{\prime \prime}=2: R(29)\right.$ and $v^{\prime}=16 \leftarrow v^{\prime \prime}=3: R_{1}$ (19), Fig. 5 (above)], produces single $P$ and $R$ emission lines, whose width is limited by the resolution of the monochromator and completely suppresses

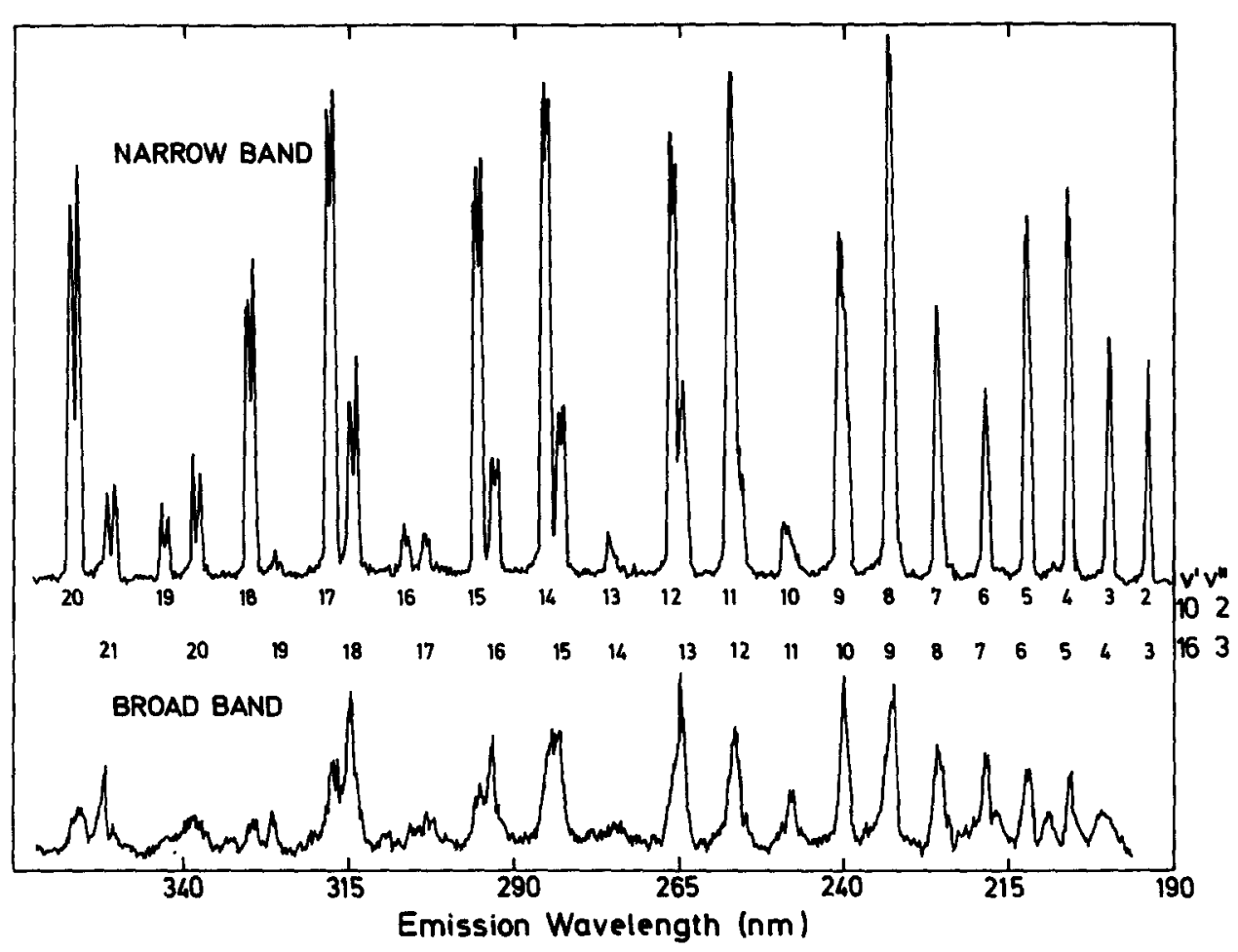

FIG. 5. Dispersion spectrum resulting from broad band laser excitation (below) and from narrow band excitation (above). Vibrational states involved in narrow band excitation are noted at far right and final vibrational states after emission are listed below the emission lines. Note small amount of NO emission in broad band spectrum. 
the NO emission. This shows clearly the absence of collisional effects even at atmospheric pressure in the fiame since collisional relaxation of the $B$ state would destroy the single $P, R$ line emission observed. At longer wavelengths the $\mathrm{O}_{2}$ emission lines split into two components, corresponding to the $v^{\prime \prime}=2$ and 3 excitations. The emission resulting from $v^{\prime \prime}=2$ excitation is always the red shifted component.

Because of the absence of quenching, very simple emission spectra arising from laser prepared single rovibronic levels are observed. Due to the large difference between the equilibrium bond lengths of the two electronic states, emission is observed to states as high as $v^{\prime \prime}=35$. Data such as that in Fig. 5 (above) were analyzed to obtain relative emission probabilities to states within $0.3 \mathrm{eV}$ of $X$-state dissociation. Great care was also taken to filter out UV emission that can appear in the monochromator's second order. The results are collected in Fig. 6.

These relative Einstein $B$ coefficients are determined by factoring out the $\boldsymbol{v}^{3}$ dependence for spontaneous emission as well as the overall experimental sensitivity as a function of

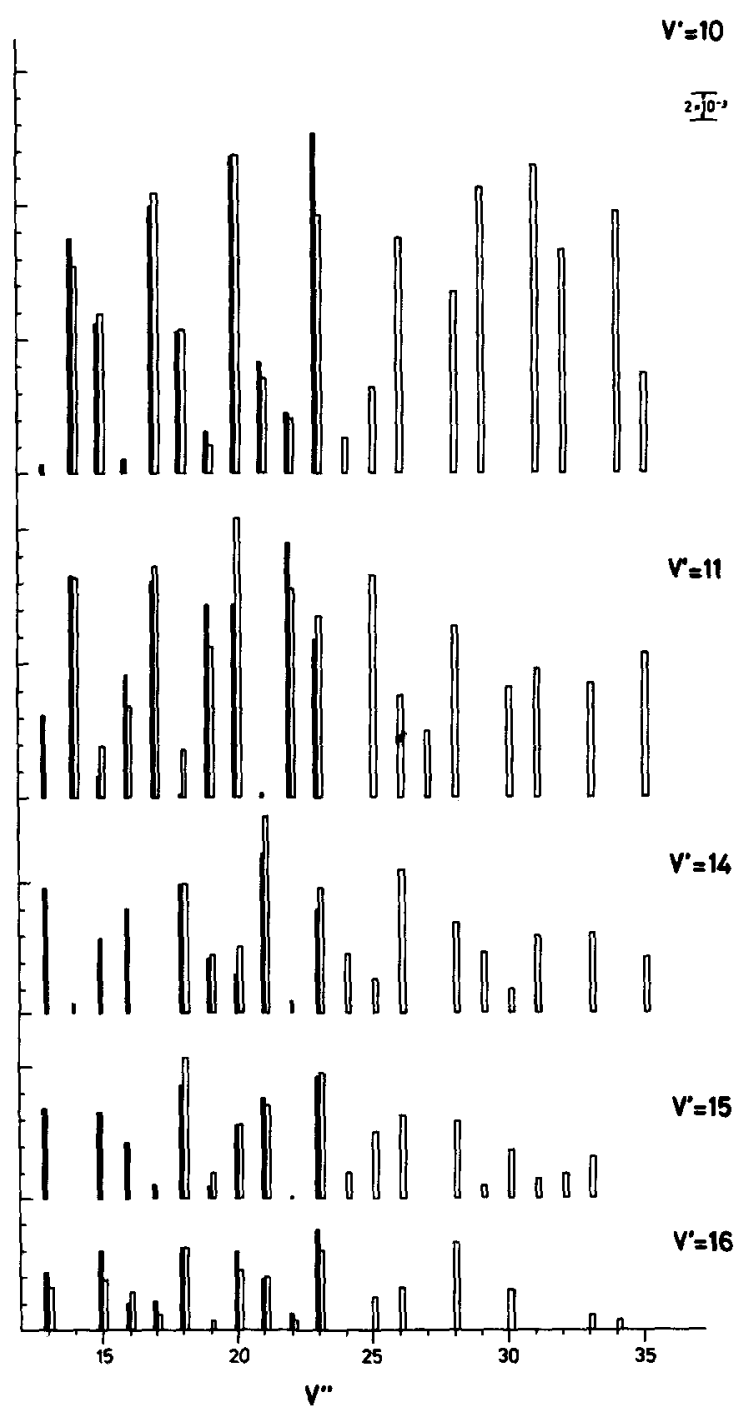

FIG. 6. Relative emission probabilities in $\mathrm{O}_{2}$. Solid lines are previously known Franck-Condon Factors from Ref. 12. the sum of the Franck-Condon factors for emission from one $v^{\prime}$ state to all $v^{\prime \prime}$ states must be unity. The Franck-Condon factor sum for $v^{\prime}=10 \rightarrow v^{\prime \prime}=0-23$, for example, is only 0.28. ${ }^{12}$ This implies that the Franck-Condon factors to states between $v^{\prime \prime}=23$ and dissociation sum to 0.62 . The experimentally derived emission probabilities in this region (shown in Fig. 6) only account for about half of this value and are therefore clearly influenced by the internuclear separation dependent transition dipole moment and cannot be simply interpreted as Franck-Condon factors.

For higher $v^{\prime \prime}$ states and consequently larger internuclear separations, transition dipole moments are not available but the variation with internuclear separation in this region is expected to be large for two reasons. First, in the separated atom limit the transition is forbidden so the transition dipole moment is going asymptotically to zero. Second, the sum of the Franck-Condon factors for emission from one $v^{\prime}$ state to all $v^{\prime \prime}$ states must be unity. The Franck-Condon factor sum for $v^{\prime}=10 \rightarrow v^{\prime \prime}=0-23$, for example, is only 0.28. ${ }^{12}$ This implies that the Franck-Condon factors to states between $v^{\prime \prime}=23$ and dissociation sum to 0.62 . The experimentally derived emission probabilities in this region (shown in Fig. 6) only account for about half of this value and are therefore clearly influenced by the internuclear separation dependent transition dipole moment and cannot be simply interpreted as Franck-Condon factors.

In general, the emission probabilities to these high vibrational states are strikingly large, especially for emission from $v^{\prime}=10,11$. Emission from $v^{\prime}=14,15$, and 16, to high vibrational states is smaller as the transition dipole moment is approaching the forbidden free-atom limit $\left(\mathrm{O}^{1} D+\right.$ $\mathrm{O}^{3} \mathrm{P} \rightarrow \mathrm{O}^{3} \mathrm{P}+\mathrm{O}^{3} \mathrm{P}$ ).

The resolution of $P$ and $R$ emission allows the rough determination of the rotational $B$ constants for these high vibrational states. Figure 7 shows the results of this analysis with a comparison to already known rotational constants. ${ }^{14}$ While the scatter in the data is relatively large, the tendency toward ever smaller $B$ values is consistent with the lengthening of the chemical bond at higher vibrational quantum numbers and further confirmation of the correct assignment of the observed emission.

\section{DISCUSSION}

\section{A. Spin-state selective predissociation in $\mathrm{O}_{2}$}

The interpretation of spin-state selective predissociation in the $\mathrm{O}_{2} B$ state depends on the spin-orbit coupling of the $B^{3} \Sigma_{u}^{-}$state to curves of differing symmetry which depends only on known spin-spin and spin-rotation splitting constants. In Hund's case A notation, such transitions must conserve the $\Omega$ quantum number (the projection of total electronic angular momentum) and parity. The critical matrix elements take the following form.

$$
\left.\mid\left\langle F_{i}\right| \mathbf{L} \cdot \text { S } \mid \text { REPULSIVE, } \Omega, \pm\right\rangle\left.\right|^{2},
$$

where: $F_{i}$ are the $B$-state triplet components, \pm is the parity of the repulsive curve. The $F_{i}$ wave functions of the $B$ state can be expressed in Hund's case A wave functions as follows. 


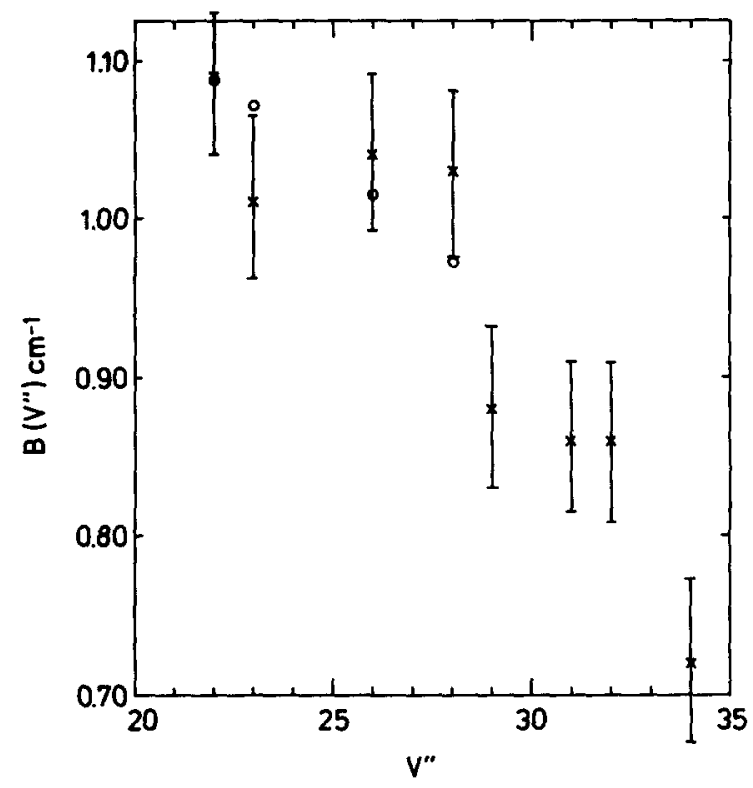

FIG. 7. Rotational $B$ constants for high vibrational states of $\mathrm{O}_{2}$.

$$
\begin{aligned}
& \left|F_{1}\right\rangle=a|\Omega=1\rangle+b|\Omega=0\rangle, \\
& \left|F_{3}\right\rangle=a|\Omega=0\rangle+b|\Omega=1\rangle, \\
& \left|F_{2}\right\rangle=|\Omega=1\rangle .
\end{aligned}
$$

The mixing coefficients are $v^{\prime}$ and $J^{\prime}$ dependent and are directly related to known spin-spin and spin-rotation constants of the $B$ state. One can see that the precise form of the matrix elements will reduce to simple functions of $a^{2}$ and $b^{2}$ and thus can be derived from experimentally known quantities. ${ }^{14}$ The dependence on repulsive state symmetry derives from the fact that each possible repulsive state has a different number of angular momentum microstates that satisfy the selection rules on $\Omega$ and parity. Such analysis does not rely on an absolute knowledge of the positions of the repulsive states or the spin-orbit coupling matrix elements, the two major sources of error in the theoretical analysis of linewidth data.

Lewis et al. have performed the most thorough analysis of absorption spectra and have attempted to determine the relative contribution to the predissociation of four repulsive states for vibrational states from $v^{\prime}=0$ to $v^{\prime}=19 .^{3}$ Such analysis suffers primarily, because it must rely on the accuracy of potential calculations for excited electronic states. The precise positions of curve crossings and the exact nature of the electronic wave functions as a function of $\mathrm{O}-\mathrm{O}$ distance determine the magnitude of the spin-orbit coupling for each repulsive curve. In contrast the analysis used to interpret relative spin-state selective predissociation rates is not influenced by the precise nature of the repulsive curves as has already been shown. Moreover, while it is sensitive to the precise nature of the repulsive curves, the rotation vibration dependence of the predissociation is not directly related to the purely electronic spin-orbit mechanism operative in this molecule. These results suggest that a more accurate theoretical treatment of the important repulsive curves is in order.

\section{B. The extension of the $\mathrm{O}_{2} \boldsymbol{X}$-state PES}

When possible diatomic PES's should be determined from spectroscopically derived molecular structure constants. Such constants for the $X$ state of $\mathrm{O}_{2}$ have been determined with reasonable accuracy up to $v^{\prime \prime}=28 .{ }^{1}$ From such information Franck-Condon factors up to $v^{\prime \prime}=23$ have been derived ${ }^{12}$ and the agreement with the results of this work is an indirect confirmation of the accuracy of the $X$ state PES up to $v^{\prime \prime}=23$. However, molecular constants of higher vibrational states are not yet available and the region of the PES between $3.7\left(v^{\prime \prime}=23\right.$ ) and $5.1 \mathrm{eV}$ (dissociation) remains undetermined. It is precisely this portion of the PES that plays a crucial role in the chemistry of $\mathrm{O}_{2}$, unimolecular dissociation and three-body recombination being the most pertinent examples. We propose that the relative emission information derived from this experiment can be used to extract the PES in this energy region.

To achieve this, one must vary an empirical $X$-state PES, calculate the Franck-Condon integrals, the dipole-moment integrals, and check against the observed emission probabilities. One must further check that the PES delivers $B$ constants that agree with those derived from the $P, R$ emission lines of this experiment, Fig. 7. Normally, this would be a very unsatisfactory method for PES determinations, because the integrals mentioned are quantities averaged over all parts of the PES. However, the Franck-Condon factors between high vibrational levels of the two electronic states result mainly from overlap of tightly localized wave functions at the outer turning point. This suggests that such data could be quite sensitive to the PES in the region of dissociation. The attractive part of the $B$-state PES is already relatively well determined up to $v^{\prime}=15$. We hope that the results of this experiment will stimulate further work in this direction.

\section{CONCLUSIONS}

Specific new results on the spectroscopy and photodissociation dynamics of $\mathrm{O}_{2}$ have been obtained by measuring LIF spectra with a tunable ArF laser. The spin-state selective predissociation has been observed in $v^{\prime}=11,14,15$, and 16 of the B-state providing a clearr picture of the mechanism of $\mathrm{O}_{2}$ predissociation for these states. The results show that the spin-state dependence of the predissociation lifetimes is a much more sensitive probe of the identities of the repulsive curves than is the vibration-rotation state dependence derived from absorption spectra. It is found that a repulsive ${ }^{3} \Sigma_{u}^{+}$state is important for high vibrational levels $\left(v^{\prime}=14,15,16\right)$. Predissociation of $v^{\prime}=11$ by a ${ }^{3} \Pi_{u}$ repulsive state was also clearly observed. These results are in direct contrast to previous studies.

By preparing single rovibronic states in high vibrational levels, simple dispersion spectra have been obtained providing relative emission probabilities to $X$-state vibrational levels within $0.3 \mathrm{eV}$ of dissociation. This data may provide the needed information to determine the $\mathrm{O}_{2}$ PES out to the dissociation limit.

'(a)K. Yoshino, D. E. Freeman, and W. H. Parkinson, J. Phys. Chem. Ref. Data 13, 207 (1984), latest review; (b) W. Lochte-Holtgreven and G. H. 
Dieke, Ann. Phys. 3, 937 (1929); (c) M. W. Feast, Proc. Phys. Soc. A 63, 549 (1950); (d) G. R. Hebert and R. W. Nichols, J. Atmos. Terrest. Phys. 21, 214 (1961); (e) L. Herman, R. Herman, and D. Rakaotaorijimy, J. Phys. Radium 22, 1 (1961); (f) M. Ogawa, Sci. Light 15, 97 (1966); (g) M. Ackerman and F. Biaume, J. Mol. Spectrosc. 35, 73 (1970); (h) J. Curry and G. Herzberg, Ann. Phys. 19, 800 (1934); (i) H. P. Krauss and S.S. Ballard, Phys. Rev. 48, 796 (1935); (j) R. D. Hudson and V. L. Carter, J. Opt. Soc. Am. 58, 1621 (1968); (k) F. Biaume, Acad. R. Belg. Mem. Cl. Sci. Collect. $8^{\circ}$ 40, 1 (1972).

2(a) D. M. Creek and R. W. Nichols, Proc. R. Soc. London Ser. A 341, 517 (1975); (b) T. H. Bergeman and S. C. Wofsy, Chem. Phys. Lett. 15, 104 (1972).

${ }^{3}$ B. R. Lewis, L. Berzins, J. H. Carver, and S. T. Gibson, J. Quant. Spectrosc. Radiat. Transfer 36, 187 (1986), and reference therein.

${ }^{4}$ H. F. Schaefer and W. H. Miller, J. Chem. Phys. 55, 4107 (1971).

(a) P. S. Julienne, J. Mol. Spectrosc. 63, 60 (1976); (b) P. S. Julienne and M. Krauss, ibid. 56, 270 (1975).
'P. Andresen, A. Bath, W. Groeger, H. W. Luelf, G. Meijer, and J. J. ter Meulen, Appl. Opt. 27, 365 (1988).

${ }^{7}$ A. M. Wodtke, L. Huwel, and P. Andresen, Rev. Sci. Instrum. (submitted).

${ }^{8}$ A. M. Wodtke, L. Huwel, H. Schluter, H. Voges, G. Meijer, and P. Andresen, 22nd Symposium (International) on Combustion, the Combustion Institute, 1988 (submitted).

${ }^{9}$ M. P. Lee and R. K. Hanson, J. Quant. Radiat. Transfer 36, 425 (1986).

${ }^{10}$ H. F. Schaefer and F. E. Harris, J. Chem. Phys. 48, 4946 (1968).

${ }^{11}$ The ArF laser is a sub-ppm detection method for NO A. M. Wodtke, $L$. Huwel, and P. Andresen, Opt. Lett. (submitted).

${ }^{12}$ R. Harris, M. Blackledge, and J. Generosa, J. Mol. Spectrosc. 30, 506 (1969).

${ }^{\text {13}}$ P. S. Julienne, D. Neumann, and M. Krauss, J. Chem. Phys. 64, 2990 (1976).

14J. B. Tatum, J. K. G. Watson, Can. J. Phys, 49, 2693 (1971). 